\title{
A common neural system mediating two different forms of social judgement
}

\author{
J. Hall ${ }^{1 *}$, H. C. Whalley ${ }^{1}$, J. W. McKirdy ${ }^{1}$, R. Sprengelmeyer ${ }^{2}$, I. M. Santos ${ }^{3}$, D. I. Donaldson ${ }^{4}$, \\ D. J. McGonigle ${ }^{5}$, A. W. Young ${ }^{6}$, A. M. McIntosh ${ }^{1}$, E. C. Johnstone ${ }^{1}$ and S. M. Lawrie $^{1}$ \\ ${ }^{1}$ Division of Psychiatry, University of Edinburgh, Edinburgh, UK \\ ${ }^{2}$ School of Psychology, University of St Andrews, St Andrews, UK \\ ${ }^{3}$ Department of Educational Sciences, University of Aveiro, Campus Universitario de Santiago, Aveiro, Portugal \\ ${ }^{4}$ Psychology Department, University of Stirling, Stirling, UK \\ ${ }^{5}$ Schools of Psychology and Biosciences, University of Cardiff, Cardiff, UK \\ ${ }^{6}$ Department of Psychology and York Neuroimaging Centre, University of York, York, UK
}

Background. A wide range of neuropsychiatric conditions, including schizophrenia and autistic spectrum disorder (ASD), are associated with impairments in social function. Previous studies have shown that individuals with schizophrenia and ASD have deficits in making a wide range of social judgements from faces, including decisions related to threat (such as judgements of approachability) and decisions not related to physical threat (such as judgements of intelligence). We have investigated healthy control participants to see whether there is a common neural system activated during such social decisions, on the basis that deficits in this system may contribute to the impairments seen in these disorders.

Method. We investigated the neural basis of social decision making during judgements of approachability and intelligence from faces in 24 healthy participants using functional magnetic resonance imaging (fMRI). We used conjunction analysis to identify common brain regions activated during both tasks.

Results. Activation of the amygdala, medial prefrontal cortex, inferior prefrontal cortex and cerebellum was seen during performance of both social tasks, compared to simple gender judgements from the same stimuli. Task-specific activations were present in the dorsolateral prefrontal cortex in the intelligence task and in the inferior and middle temporal cortex in the approachability task.

Conclusions. The present study identified a common network of brain regions activated during the performance of two different forms of social judgement from faces. Dysfunction of this network is likely to contribute to the broad-ranging deficits in social function seen in psychiatric disorders such as schizophrenia and ASD.

Received 21 March 2009; Revised 20 August 2009; Accepted 20 August 2009; First published online 8 October 2009

Key words: Amygdala, faces, fMRI, prefrontal cortex, social.

\section{Introduction}

A wide range of neuropsychiatric disorders are associated with impairments in social cognition. Particularly prominent deficits in social interaction are seen in schizophrenia and autistic spectrum disorder (ASD). Impaired social function is a key diagnostic feature of schizophrenia in the DSM-IV classification, and has been shown to relate to long-term prognosis (APA, 1994). Similarly, difficulties with social interactions and communication are part of the core pathology of

* Address for correspondence: Dr J. Hall, Division of Psychiatry, University of Edinburgh, Kennedy Tower, Royal Edinburgh Hospital, Edinburgh EH10 5HF, UK.

(Email: jhall5@staffmail.ed.ac.uk) autism and account for much of the disability associated with the disorder (APA, 1994).

Facial expressions are a major cue used in social interactions (Darwin, 1872; Haxby et al. 2002; Adolphs, 2003). Behavioural studies have demonstrated deficits in social judgement from faces in schizophrenia and ASD. The majority of studies have investigated the ability of affected individuals to identify basic emotional expressions from faces. Patients with schizophrenia have been shown to have deficits in the recognition of negative facial emotions, especially during psychotic episodes (Mandal et al. 1998; Edwards et al. 2002; Marwick \& Hall, 2008). Individuals with ASD have also been shown to have impairments in facial emotion recognition, with some studies finding a particular deficit for the emotion of fear whereas other studies report a more pervasive 
deficit (Hobson et al. 1988; Celani et al. 1999; Howard et al. 2000; Adolphs et al. 2001; Pelphrey et al. 2002).

Fewer studies have investigated the ability of individuals with schizophrenia and autism to make more complex social judgements from faces. The available studies have focused on decisions related to threat, particularly judgements of approachability and trustworthiness from faces. There is evidence that approachability and trustworthiness judgements are abnormal in schizophrenia, an effect that may be more pronounced in paranoid individuals (Hall et al. 2004; Baas et al. 2008b; Pinkham et al. 2008). Similarly, individuals with ASD have also been shown to have impairments in rating approachability and trustworthiness from faces (Adolphs et al. 2001), and in labelling complex emotions from images of eyes (Baron-Cohen et al. 2001).

In previous work we have demonstrated deficits in patients with schizophrenia and ASD in making social judgement from faces using a battery of tests covering a range of different social dimensions (Hall et al. 2004; Philip et al. in press). Patients with both disorders showed deficits in social judgement that were not restricted to affective, threat-related decisions (such as approachability) but extended to judgements of intelligence and distinctiveness from faces (Hall et al. 2004; Philip et al. in press). These results suggest that a common system underlying a wide range of social judgements from faces is disrupted in both schizophrenia and ASD (Brothers, 1990; Haxby et al. 2000, 2002; Adolphs, 2003; Brunet-Gouet \& Decety, 2006; Amaral et al. 2008; Pinkham et al. 2008).

Previous imaging studies of social cognition have implicated several brain regions in social decision making, including the amygdala, medial prefrontal cortex, orbitofrontal cortex and lateral temporal cortex (Winston et al. 2002; Adolphs, 2003; Amodio \& Frith, 2006; Winston et al. 2007; Baas et al. 2008a). The amygdala in particular has been noted to be activated in relation to potential social threat, and has been hypothesized to act as a reflexive monitor of danger (Dolan \& Vuilleumier, 2003). However, few studies have investigated the neural basis of less overtly threat-related social decisions, such as judgements of intelligence from faces. To our knowledge, no previous studies have directly compared brain activation during more than one test of social judgement.

In the current study we investigated the neural basis of social judgements for both overtly affective, threat-related social judgements (judgements of approachability) and social judgements that do not relate directly to the evaluation of physical threat (judgements of intelligence), both of which are impaired in schizophrenia and ASD (Hall et al. 2004; Philip et al. in press). We investigated the basis of such judgements in healthy control subjects to help elucidate the neural basis of the cognitive function disturbed in neuropsychiatric disorders such as schizophrenia and ASD, while avoiding the potential confounds of scanning studies of individuals with these disorders (such as differential task performance). We hypothesized that a common set of brain regions would be required for both types of social decision, impairments in which are likely to underlie the deficits seen in social cognition in schizophrenia and autism (Brothers, 1990; Brunet-Gouet \& Decety, 2006; Amaral et al. 2008).

\section{Method}

\section{Participants}

Twenty-four right-handed volunteers participated in the study [12 males, 12 females; mean age 29.3 (S.D. = 8.3) years; mean IQ 115.3 (s.D.=5.6)]. Exclusion criteria included a history of neurological or psychiatric disorder. All participants gave informed consent as approved by the Local Research Ethics Committee.

\section{Experimental design}

Two tests of social cognition were performed comprising judgements of approachability or intelligence from faces (Hall et al. 2004; Santos \& Young, 2008). In the approachability task, participants had to decide whether faces appeared 'not approachable' or 'very approachable'. In the intelligence task, participants had to decide whether the faces appeared 'not intelligent' or 'very intelligent'. The control condition for each task consisted of categorically rating gender from the same faces, with the stimuli used for this and the main task being counterbalanced across participants.

Facial stimuli were selected as described previously (Hall et al. 2004; Santos \& Young, 2008). In brief, 1000 pictures of faces derived from media sources, all of non-famous adults, were shown to six volunteer participants and were rated for approachability and intelligence on a scale of 1-7. The faces were highly reliably rated on both social dimensions across all raters $(p<0.01$, Cronbach's $\alpha=0.79$ for approachability judgements and 0.75 for intelligence judgements). Faces representing the extremes of each social dimension were selected as stimuli for the neuroimaging task. Notably, there was a low overall correlation (0.26) between decisions made on the approachability and intelligence judgement tasks, suggesting that these tasks test different dimensions of social judgement (Santos, 2003).

Two sets of facial stimuli (A and B) were assembled for each task. The sets consisted of 18 male and 18 
female faces each. The faces of each sex were selected to maximize the difference across each social dimension examined (for example, in the approachability condition, nine high approachability faces and nine low approachability faces of each gender per set). For each participant one set of faces was used for social judgements and the other set of faces was used for gender judgements. The use of the stimulus sets was counterbalanced across participants such that half the participants made social judgements from stimulus set $A$ and control gender judgements from stimulus set $B$ and half the participants made social judgements from stimulus set $\mathrm{B}$ and control gender judgements from stimulus set $\mathrm{A}$.

Both social judgement tasks (approachability and intelligence) were constructed to consist of two runs of six blocks per run. For each task, blocks of the social judgement ('Social' condition) were alternated with blocks of gender judgement ('Gender' condition) and the order of the blocks was counterbalanced across participants. Each block was $25 \mathrm{~s}$ in duration and blocks were separated by a rest period of $12.5 \mathrm{~s}$ during which participants were instructed to fixate on a cross in the centre of the screen ('Rest' condition). Blocks commenced with a $1 \mathrm{~s}$ visual prompt of the nature of the task to be performed (e.g. 'Approachability'). Six faces were then presented in each block. Each face was presented for $3.5 \mathrm{~s}$ separated by a $0.5 \mathrm{~s}$ interstimulus interval (ISI). Faces were presented in one of four fixed pseudo-random orders, counterbalanced across participants, with the constraint that no more than three faces of one end of the dimension should be presented sequentially. The alternative response choices were shown on the screen throughout the task block (e.g. 'not approachable' and 'very approachable') and participants had to press one of two buttons to indicate which response they felt was most appropriate for each face shown. Participants were able to respond at any time during the $3.5 \mathrm{~s}$ face presentation or during the subsequent $0.5 \mathrm{~s}$ ISI. Responses on the social judgement tests were scored according to their agreement with the response most commonly selected in the ratings study, with a maximum score of 36 in each category. Reaction times were recorded for all judgements made in the scanner. Behavioural data from the scanning session were unavailable for one participant due to technical error; however, the participant reported completing the task and this was confirmed by real-time behavioural monitoring during the scanning session and therefore imaging data from this participant were included. The overall order of tasks (intelligence or approachability) was counterbalanced across participants.

Participants were instructed in how to perform the task prior to the commencement of testing and were given a short practice version of the tasks, consisting of a block of each judgement.

\section{Image acquisition}

Imaging was performed at the SFC Brain Imaging Research Centre in Edinburgh using a GE 1.5 T Signa scanner (GE Medical, USA). After a localizer scan, participants underwent four functional scanning runs [two runs each of approachability and intelligence tasks; 99 volumes/session; field of view $22 \mathrm{~cm}$; echo time (TE) $40 \mathrm{~ms}$; repetition time (TR) $2.5 \mathrm{~s}]$. Interleaved axial slices were acquired with a thickness of $5 \mathrm{~mm}$ and a matrix size of $64 \times 64$. The first four echoplanar images (EPIs) in each run were discarded to avoid T1 equilibrium effects.

\section{Image processing and analysis}

The EPIs were reconstructed offline in ANALYZE format (Mayo Foundation, USA). Image analysis was conducted using SPM2 (Statistical Parametric Mapping; Wellcome Department of Cognitive Neurology and collaborators, Institute of Neurology, London, UK). Pre-processing consisted of re-orientation of the images and realignment to the mean EPI image, followed by normalisation to the standard Montreal Neurological Institute (MNI) EPI template and spatial smoothing using a Gaussian kernel $\left(8 \mathrm{~mm}^{3}\right.$ full-width at half-maximum). The participant's data were filtered in time using a high-pass filter (150 s cut-off) and temporal autocorrelations were accounted for by using an AR(1) model.

Statistical analysis was performed using the general linear model approach as implemented in SPM2. At the individual participant level the data for each task were modelled with three conditions (Social, Gender and Rest), each modelled by a boxcar convolved with a canonical haemodynamic response function. Parameters representing the participants' movement during the scan were also entered into the model as covariates of no interest. Contrast images were generated for each participant for the principal contrast of interest (Social versus Gender) representing the pairwise comparison of parameter estimates for the conditions. One contrast image per participant was then entered into a second-level random effects analysis to examine regions of significant activation across the group using a one-sample $t$ test.

A conjunction analysis was performed to determine which areas showed common activation across the two social cognition tasks. This is equivalent to a logical AND function. For the conjunction analysis a one-way ANOVA was constructed with task as the grouping variable and one contrast image per task was 
Table 1. Brain regions activated during judgements of approachability and intelligence from faces

\begin{tabular}{|c|c|c|c|c|}
\hline$p_{\text {corr }}$ & $K_{\mathrm{E}}$ & Peak $T$ & Coordinates (MNI) & Location of peak voxel \\
\hline \multicolumn{5}{|c|}{ Approachability judgements versus gender judgements } \\
\hline$<0.001$ & 4852 & 7.02 & $-8,56,44$ & L Medial prefrontal \\
\hline$<0.001$ & 1461 & 7.52 & $-30,18,-24$ & L Inferior frontal gyrus \\
\hline$<0.001$ & 856 & 5.78 & $50,-6,-34$ & R Inferior temporal gyrus \\
\hline 0.002 & 525 & 4.61 & $-52,-24,-12$ & L Middle temporal gyrus \\
\hline$<0.001$ & 874 & 5.17 & $30,-88,-38$ & R Cerebellum \\
\hline$<0.001$ & 1393 & 6.59 & $-24,-90,-38$ & L Cerebellum \\
\hline $0.029^{\mathrm{a}}$ & 23 & 3.96 & $18,2,-18$ & R Amygdala \\
\hline $0.035^{\mathrm{a}}$ & 17 & 3.88 & $-22,-8,-20$ & L Amygdala \\
\hline \multicolumn{5}{|c|}{ Intelligence judgements versus gender judgements } \\
\hline$<0.001$ & 6479 & 9.05 & $-10,20,54$ & L Medial prefrontal \\
\hline$<0.001$ & 2877 & 8.35 & $-50,28,2$ & L Inferior frontal gyrus \\
\hline$<0.001$ & 2388 & 9.37 & $52,18,42$ & $\begin{array}{l}\text { R Dorsolateral prefrontal } \\
\text { (extending to R inferior frontal gyrus) }\end{array}$ \\
\hline$<0.001$ & 1259 & 6.37 & $-10,14,10$ & $\mathrm{~L}$ and $\mathrm{R}$ Caudate nucleus \\
\hline 0.005 & 306 & 5.58 & $0,-18,-22$ & Peri-aqueductal grey \\
\hline$<0.001$ & 6274 & 9.19 & $-48,-66,-34$ & L Cerebellum (extending to R cerebellum) \\
\hline $0.007^{\mathrm{a}}$ & 61 & 4.80 & $20,-2,-16$ & R Amygdala \\
\hline $0.008^{\mathrm{a}}$ & 67 & 4.86 & $-14,-6,-18$ & L Amygdala \\
\hline
\end{tabular}

L, Left; R, right.

${ }^{a}$ Within a bilateral amygdala small volume correction (SVC).

entered into each group for each participant. $t$ tests were conducted to determine the main effects of each task and conjoint activation across the tasks was determined by inclusive masking of the main effects of one task with the other at a threshold of $p<0.001$. Identical results were also obtained using the procedure described by Nichols et al. (2005).

All statistical maps were thresholded at a level of $p<0.001$ uncorrected and regions were considered significant at $p<0.05$ at the cluster level, corrected for multiple comparisons (cluster correction across whole brain volume as implemented in SPM2). Region of interest (ROI) analysis was conducted for the bilateral amygdala using a small volume correction (SVC) derived from the automated anatomical labelling atlas in WFU_PickAtlas v.2.0 dilated by 1 voxel to incorporate the full extent of the amygdala complex (TzourioMazoyer et al. 2002; Maldjian et al. 2003).

\section{Results}

\section{Behavioural data}

Behavioural data were recorded from participants as they completed the tasks in the scanner. Responses on both tasks showed a high degree of reproducibility across participants. The mean scores (out of 36) on the approachability task were 31.5 (S.D. $=4.1$ ) for social judgements and 34.8 (S.D.=1.0) for gender judgements. The mean scores on the intelligence task were
29.3 (s.D. = 3.7) for social judgements and 35.1 (s.D.= 1.0) for gender judgements. There was no significant difference in performance between the approachability and intelligence tasks $(p>0.05)$. Participants performed the gender judgements more accurately than the social judgements in both tasks $(p<0.01$ in both cases). The mean reaction times (RTs) during the approachability task were $1316 \mathrm{~ms}$ (S.D. =237) for social judgements and $1056 \mathrm{~ms}$ (S.D. =182) for gender decisions. The mean RTs during the intelligence task were $1550 \mathrm{~ms}$ (S.D. $=237$ ) for social judgements and 1072 ms (S.D. =182) for gender decisions. Analysis of the RT data revealed that gender judgements were performed more quickly than social judgements in both tasks ( $p<0.001$ in both cases), with no difference in the RTs for gender judgements between the two tasks $(p>0.8)$. Approachability judgements were made significantly more quickly than judgements of intelligence $(p=0.003)$.

\section{Neural responses during judgements of approachability from faces}

To investigate neural responses related to judgements of approachability, we compared blood oxygen leveldependent (BOLD) activations during approachability to those during judgements of gender [Table 1 and Figs 1(a) and 2]. Notably, the two stimulus sets were counterbalanced across subjects, such that half the subjects made approachability judgements on 
(a)

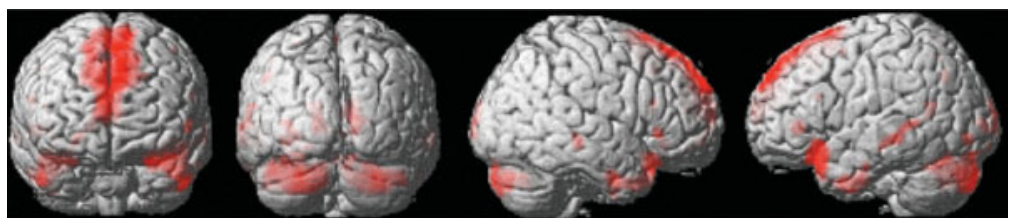

(b)

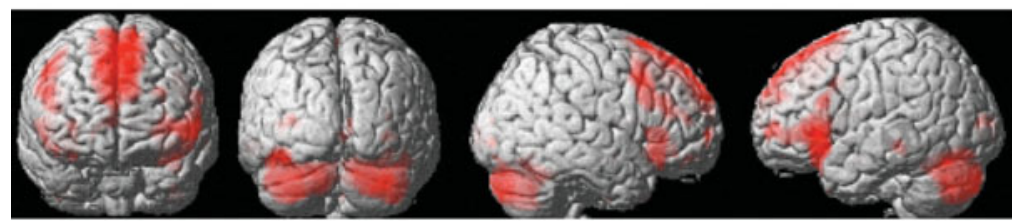

(c)

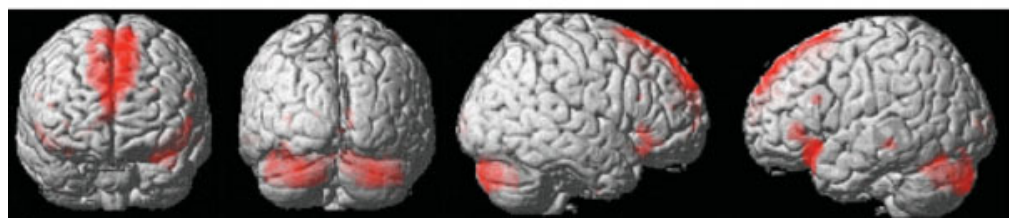

Fig. 1. Brain regions activated during social judgement. Statistical maps of task activations rendered on a whole brain image showing: (a) approachability judgements versus gender judgements, $(b)$ intelligence judgements versus gender judgements and (c) conjunction of approachability and intelligence judgements. Images thresholded at $p<0.001$ uncorrected.
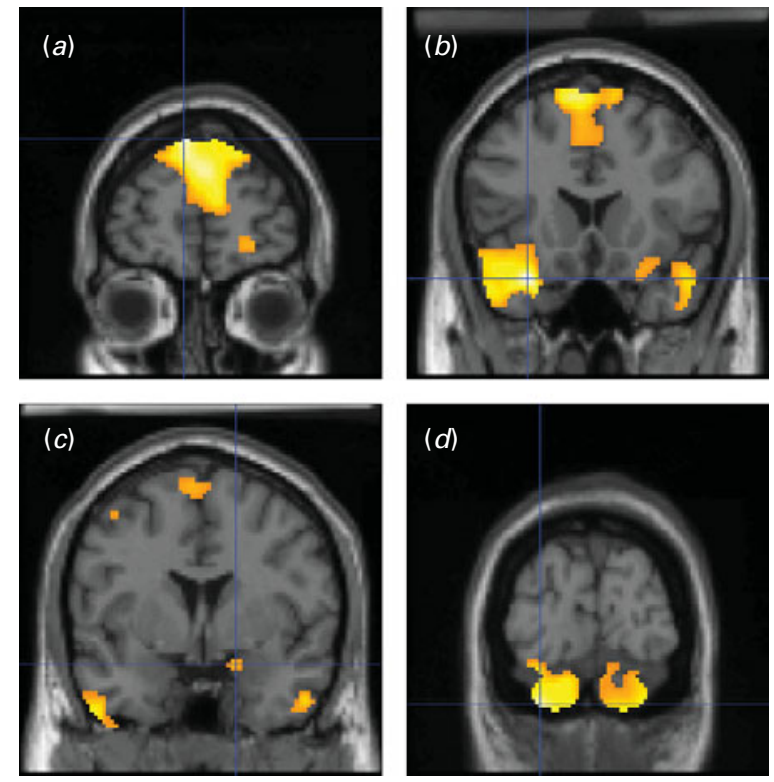

Fig. 2. Statistical parametric maps (SPMs) showing peak activations during approachability judgements in $(a)$ left medial prefrontal cortex, $(b)$ left inferior prefrontal cortex,

(c) right amygdala and (d) left cerebellum. SPMs thresholded at $p<0.001$.

stimulus set $\mathrm{A}$ and gender judgements on stimulus set $B$ and the other half made gender judgements on stimulus set A and approachability judgements in stimulus set $\mathrm{B}$. The contrast of approachability judgements versus gender judgements revealed significant task-related activations in the anterior and superior medial prefrontal cortex bilaterally [Brodmann area (BA) 6 and BA 9], with the peak activation seen on the left. In addition, bilateral activation of the inferior frontal gyrus was seen extending to the insula (BA 45/ 47), which on the left formed part of a contiguous cluster extending into the inferior temporal cortex. Bilateral activation was present in the posterior cerebellum and the inferior temporal gyrus (BA 20) extending into the temporal poles. Unilateral activation was demonstrated in the left middle temporal gyrus (BA 21). Bilateral activation of the amygdala was observed during approachability judgements (compared to gender judgements), which reached corrected significance within an anatomically defined ROI.

\section{Neural responses during judgements of intelligence from faces}

We next investigated brain activation during judgements of intelligence compared to judgements of gender from matched stimuli (Table 1 and Figs $1 b$ and 3). This contrast revealed significant bilateral activation in the dorsal medial prefrontal cortex (BA 6) extending into the rostral prefrontal cortex (BA 9) with more prominent activation on the left side. Bilateral activation was also seen in the inferior frontal gyrus extending posteriorly to the insula on the left (BA 45/47) and in the posterior cerebellum. No activation was seen in the inferior or middle temporal regions. However, activation was seen in the right dorsolateral prefrontal cortex (BA 9) and bilaterally in the caudate nucleus, areas that were not active during approachability judgements. In addition, there was a significant cluster of activation extending from the midbrain in the region of the peri-aqueductal grey through the amygdala bilaterally and incorporating part of the hypothalamus. 

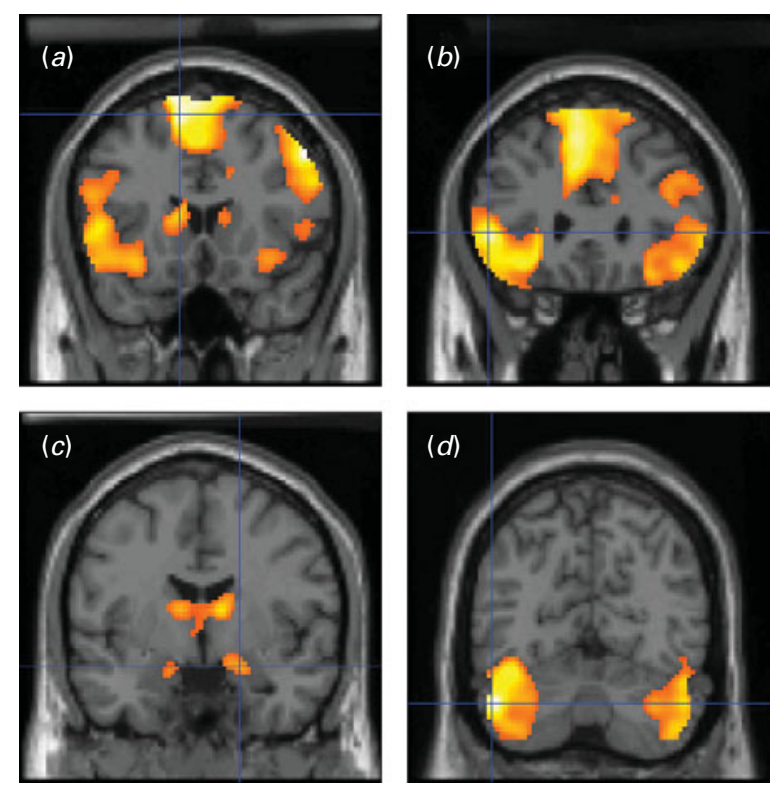

Fig. 3. Statistical parametric maps (SPMs) showing peak activations during intelligence judgements in (a) left medial prefrontal cortex, $(b)$ left inferior prefrontal cortex, $(c)$ right amygdala and $(d)$ left cerebellum. SPMs thresholded at $p<0.001$.

\section{Conjunction analysis}

We next investigated whether there was a common network of brain regions showing task-related activation in both the approachability and intelligence tasks. To do this we conducted a conjunction analysis to produce a statistical map of voxels activated at $p<0.001$ uncorrected in both tasks. We then used this map to identify clusters showing significant activation across the two tasks with a corrected cluster significance of $p<0.05$. In addition, we looked for conjunctional activation of the amygdala across the two tasks at cluster $p<0.05$ corrected within a bilateral amygdala ROI.

Areas showing a significant conjunction of activation across the two tasks are shown in Table 2 and Fig. 1c. Common activation was seen in the superior and anterior medial prefrontal cortex (BA 6 and BA 9), bilateral inferior frontal cortex (BA 45/47) extending into the insula on the left, and bilateral posterior cerebellum. ROI analysis also identified significant cluster-level conjunctional activation in both the left and right amygdala.

\section{Discussion}

Impairments in social cognition are major features of psychiatric disorders including schizophrenia and ASD. We have previously shown that both schizophrenia and ASD are associated with impairments in making a wide range of social judgements, including judgements of approachability and intelligence from faces, using the same tasks behaviourally as used in the current study (Hall et al. 2004; Philip et al. in press). Here we have used functional magnetic resonance imaging (fMRI) to investigate whether there is a common neural system underlying such social judgements in health, impairments in which could account for the deficits seen in these disorders. Our results confirm that there is a common set of brain regions activated during judgements of both approachability and intelligence from faces that includes the amygdala, medial prefrontal cortex, inferior prefrontal cortex and cerebellum.

Bilateral amygdala activation was seen in both tasks. The amygdala has previously been implicated in social judgement, especially for tasks with an explicitly affective nature (Adolphs, 2003; Adolphs \& Spezio, 2006). Lesions of the amygdala result in impairments in judgements of trustworthiness and approachability, and amygdala activation has been demonstrated in functional imaging tasks to faces rated as untrustworthy (Adolphs et al. 1998; Winston et al. 2002). Studies of facial emotion processing have also demonstrated a central role of the amygdala in detecting negative emotions such as fear in faces (Adolphs et al. 1994, 1999; Breiter et al. 1996; Morris et al. 1996). Taken together, these results have led to the suggestion that the role of the amygdala in social judgement is to act as an implicit detector of threat or hostility (Dolan \& Vuilleumier, 2003). The current findings, however, support a broader involvement of the amygdala in social judgement. Activation of the amygdala was seen not only in the approachability task, which is clearly threat related in nature, but also during the intelligence judgement task, which is not primarily related to threat. In addition, greater amygdala activation was seen during social judgements than during gender judgements from the same faces (across subjects), indicating a role of the amygdala in social judgement that extends beyond automatic responding to features of facial stimuli related to threat (Baron-Cohen et al. 1999). These results are consistent with a general role of the amygdala in inferring others' mental states (the so-called 'theory of mind') (Kling \& Brothers, 1992; Baron-Cohen et al. 1999; Fine et al. 2001), a view supported by lesion studies showing that amygdala damage results in impairments in a wide range of social judgements from faces (Adolphs et al. 1998; Adolphs et al. 2002; Stone et al. 2003; Shaw et al. 2005) and deficits in non-facial theory of mind tasks (Fine et al. 2001; Stone et al. 2003).

The medial prefrontal cortex, particularly on the left, has been shown to be activated in tasks testing social decision making and theory of mind 
Table 2. Brain regions showing significant activation in both social judgement tasks as assessed by conjunction analysis

\begin{tabular}{|c|c|c|c|c|}
\hline$p_{\text {corr }}$ & $K_{\mathrm{E}}$ & Peak $T$ & Coordinates & Location of peak activation \\
\hline \multicolumn{5}{|c|}{ Areas showing significant activation in conjunction analysis } \\
\hline$<0.001$ & 4717 & 6.71 & $-10,16,66$ & L Medial prefrontal - dorsal (BA 6) \\
\hline$<0.001$ & 943 & 5.71 & $-40,22,-14$ & L Inferior frontal gyrus (BA 45/47) \\
\hline 0.003 & 477 & 4.11 & $46,24,-16$ & R Inferior frontal gyrus (BA 45/47) \\
\hline$<0.001$ & 2957 & 5.62 & $32,-86,-40$ & $\mathrm{~L}$ and $\mathrm{R}$ cerebellum \\
\hline \multicolumn{5}{|c|}{ Amygdala ROI } \\
\hline 0.030 & 21 & 3.80 & $-20,-8,-20$ & L Amygdala \\
\hline 0.044 & 10 & 3.74 & $18,0,-16$ & R Amygdala \\
\hline
\end{tabular}

L, Left; R, right; BA, Brodmann area; ROI, region of interest.

judgements (Amodio \& Frith, 2006; Brunet-Gouet \& Decety, 2006), leading to the suggestion that this brain region may have a central role in forming higher-level representations about the intentions of others (Amodio \& Frith, 2006). Meta-analyses of neuroimaging studies have confirmed the involvement of the medial prefrontal cortex in emotional and social tasks and have suggested that more rostral regions of the medial prefrontal cortex may be preferentially involved in 'affective' tasks whereas more dorsal regions may be selectively activated during more 'cognitive' processing (Bush et al. 2000; Steele \& Lawrie, 2004). In this regard it is of interest that the peak activation in the intelligence judgement task was more dorsal than that in the approachability task. Conjunction analysis, however, revealed that there was considerable overlap in the regions of the medial prefrontal cortex activated in the two tasks in the current study, demonstrating that a core region of medial prefrontal cortex is activated across different social judgements.

The inferior prefrontal cortex and anterior insula were activated in both tasks and have been shown previously to operate as part of the mirror neuron system (Gallese et al. 2004; Rizzolatti \& Craighero, 2004). The pars opercularis is recruited during the execution of an action and the observation of the same action in others, whereas the pars orbitalis and the insula have been shown to play a similar role in representing emotional states in the self and others (Craig, 2002; Decety \& Chaminade, 2003; Gallese et al. 2004; Singer et al. 2004). Mirror activation in these brain regions is thought to underlie the generation of an internal state in the observer similar to that present in the observed subject (Carr et al. 2003; Gallese et al. 2004). Activation of these brain regions has been seen in social judgement tasks (Baron-Cohen et al. 1999; Russell et al. 2000) and may reflect the generation of a subjective representation of the affective state of others used to guide decision making (Gallese et al. 2004).
The posterior lobe of the cerebellum showed bilateral activation in both tests of social cognition in the current study and has previously been implicated in theory of mind judgements (Brunet et al. 2000; Calarge et al. 2003). Lesions to this brain region result in the cerebellar cognitive affective syndrome, which includes deficits in executive function, personality changes and alterations in social function including inappropriate behaviour (Schmahmann \& Sherman, 1998; Schmahmann, 2004). The posterior cerebellum has extensive reciprocal connections through the pons to prefrontal, temporal and limbic regions and may play a role in the coordination of higher cognitive function including social judgement (Schmahmann, 2004).

A more limited set of brain regions showed selective activation in only one of the social judgement tasks tested. Activation of the right dorsolateral prefrontal cortex was seen only in the intelligence judgement task. An increased BOLD signal was also seen in the intelligence task in the head of the caudate nucleus, the striatal projection area of the dorsolateral prefrontal cortex. These regions may represent a functional circuit recruited during social judgements of a more cognitive and less affective nature. Lesions of the dorsolateral prefrontal cortex have been shown to result in impairments in the ability of subjects to use social cues to make interpersonal judgements, supporting a functional role of the dorsolateral prefrontal cortex in some forms of social decision making (Mah et al. 2004). Activation of the dorsolateral prefrontal cortex and caudate nucleus may also relate to the overall cognitive load, as the RT data indicate that the intelligence judgement task is more cognitively demanding than the approachability task. By contrast, activation of the inferior temporal cortices extending to the temporal pole and the left middle temporal cortex was only seen in the approachability task. Temporal lobe regions, including the temporal poles and middle and inferior temporal cortices, have been implicated in theory of mind judgements and in 
assessing and empathizing with facial affect (Carr et al. 2003; Gallagher \& Frith, 2003; Kim et al. 2005; Vollm et al. 2006). Activity in these regions may therefore be required to access mnemonic information used in social judgement, especially in relation to decisions of an affective nature.

The present study represents a large neuroimaging investigation of the neural basis of social judgements; however, some limitations of this study should be noted. First, we used a blocked design comparing social judgements to gender judgements, comparable to tasks in which we have previously shown patient groups to be impaired (Hall et al. 2004; Philip et al. in press). Although this is a statistically powerful method, the design of the task did not enable us to separately investigate stimulus- and task-driven neural responses, or the interaction between these factors. A fuller analysis of these features would require an event-related or mixed blocked and eventrelated design as used in some previous investigations of social judgement (Winston et al. 2002, 2007; Baas et al. 2008a). Second, although a strength of the current study was the investigation of two different social judgements, practical limitations prevented the investigation of the neural basis of a broader range of social decisions. Third, we cannot entirely exclude the possibility that judgements of intelligence are also to some degree threat related, although previous evidence suggests that there is only a very low correlation between performance on approachability and intelligence judgements (Santos, 2003). Fourth, the gender judgements used as the comparison condition themselves represent a form of social judgement; however, gender judgements were performed uniformly more accurately and rapidly than social judgements in the current study, confirming that they represent a constrained but cognitively less demanding control condition. These caveats notwithstanding, the identification of a common brain network involved in both tasks strongly implicated abnormalities in these brain regions, or their coordinated interaction, in the pathogenesis of deficits in social cognition in neuropsychiatric disorders such as schizophrenia and ASD and other conditions in which social deficits feature prominently, including personality disorders (BaronCohen et al. 1999; Pinkham et al. 2003; Abdi \& Sharma, 2004; Brunet-Gouet \& Decety, 2006; Amaral et al. 2008).

\section{Acknowledgements}

The work was funded by the Dr Mortimer and Theresa Sackler Foundation. J.H. is supported by a Medical Research Council (MRC) Clinical Research Training Fellowship and A.McI. is supported by The Health
Foundation. H.C.W. and S.M.L. were supported by the Sackler Foundation. Imaging was performed at the SFC Brain Imaging Research Centre in Edinburgh. We thank all the participants.

\section{Declaration of Interest}

J.H., A.McI., H.C.W. and S.M.L. have received grants from the Translational Medicine Research Collaboration, a consortium made up of the Universities of Aberdeen, Dundee, Edinburgh and Glasgow, the four associated National Health Service (NHS) Health Boards (Grampian, Tayside, Lothian and Greater Glasgow \& Clyde), Scottish Enterprise, and Wyeth Pharmaceuticals.

\section{References}

Abdi Z, Sharma T (2004). Social cognition and its neural correlates in schizophrenia and autism. CNS Spectrums $\mathbf{9}$, 335-343.

Adolphs R (2003). Cognitive neuroscience of human social behaviour. Nature Reviews Neuroscience 4, 165-178.

Adolphs R, Baron-Cohen S, Tranel D (2002). Impaired recognition of social emotions following amygdala damage. Journal of Cognitive Neuroscience 14, 1264-1274.

Adolphs R, Sears L, Piven J (2001). Abnormal processing of social information from faces in autism. Journal of Cognitive Neuroscience 13, 232-240.

Adolphs R, Spezio M (2006). Role of the amygdala in processing visual social stimuli. Progress in Brain Research 156, 363-378.

Adolphs R, Tranel D, Damasio AR (1998). The human amygdala in social judgment. Nature 393, 470-474.

Adolphs R, Tranel D, Damasio H, Damasio A (1994). Impaired recognition of emotion in facial expressions following bilateral damage to the human amygdala. Nature 372, 669-672.

Adolphs R, Tranel D, Hamann S, Young AW, Calder AJ, Phelps EA, Anderson A, Lee GP, Damasio AR (1999). Recognition of facial emotion in nine individuals with bilateral amygdala damage. Neuropsychologia 37, 1111-1119.

Amaral DG, Schumann CM, Nordahl CW (2008). Neuroanatomy of autism. Trends in Neurosciences 31, 137-145.

Amodio DM, Frith CD (2006). Meeting of minds: the medial frontal cortex and social cognition. Nature Reviews Neuroscience 7, 268-277.

APA (1994). Diagnostic and Statistical Manual of Mental Disorders, 4th edn. American Psychiatric Press: Washington, DC.

Baas D, Aleman A, Vink M, Ramsey NF, de Haan EHF, Kahn RS (2008a). Evidence of altered cortical and amygdala activation during social decision-making in schizophrenia. NeuroImage 40, 719-727.

Baas D, van't Wout M, Aleman A, Kahn RS (2008b). Social judgement in clinically stable patients with schizophrenia 
and healthy relatives: behavioural evidence of social brain dysfunction. Psychological Medicine 38, 747-754.

Baron-Cohen S, Ring HA, Wheelwright S, Bullmore ET, Brammer MJ, Simmons A, Williams SC (1999). Social intelligence in the normal and autistic brain: an fMRI study. European Journal of Neuroscience 11, 1891-1898.

Baron-Cohen S, Wheelwright S, Hill J, Raste Y, Plumb I (2001). The 'Reading the Mind in the Eyes' Test revised version: a study with normal adults, and adults with Asperger syndrome or high-functioning autism. Journal of Child Psychology and Psychiatry 42, 241-251.

Breiter HC, Etcoff NL, Whalen PJ, Kennedy WA, Rauch SL, Buckner RL, Strauss MM, Hyman SE, Rosen BR (1996). Response and habituation of the human amygdala during visual processing of facial expression. Neuron 17, 875-887.

Brothers L (1990). The social brain: a project for integrating primate behaviour and neurophysiology in a new domain. Concepts in Neuroscience 1, 27-51.

Brunet E, Sarfati Y, Hardy-Bayle M-C, Decety J (2000). A PET investigation of the attribution of intentions with a nonverbal task. NeuroImage 11, 157-166.

Brunet-Gouet E, Decety J (2006). Social brain dysfunctions in schizophrenia: a review of neuroimaging studies. Psychiatry Research: Neuroimaging 148, 75-92.

Bush G, Luu P, Posner MI (2000). Cognitive and emotional influences in anterior cingulate cortex. Trends in Cognitive Sciences 4, 215-222.

Calarge C, Andreasen NC, O'Leary DS (2003). Visualizing how one brain understands another: a PET study of theory of mind. American Journal of Psychiatry 160, 1954-1964.

Carr L, Iacoboni M, Dubeau M-C, Mazziotta JC, Lenzi GL (2003). Neural mechanisms of empathy in humans: a relay from neural systems for imitation to limbic areas. Proceedings of the National Academy of Sciences USA 100, 5497-5502.

Celani G, Battacchi MW, Arcidiacono L (1999). The understanding of the emotional meaning of facial expressions in people with autism. Journal of Autism and Developmental Disorders 29, 57-66.

Craig AD (2002). How do you feel? Interoception: the sense of the physiological condition of the body. Nature Reviews Neuroscience 3, 655-666.

Darwin C (1872). The Expression of the Emotions in Man and Animals. John Murray: London.

Decety J, Chaminade T (2003). Neural correlates of feeling sympathy. Neuropsychologia 41, 127-138.

Dolan RJ, Vuilleumier P (2003). Amygdala automaticity in emotional processing. Annals of the New York Academy of Sciences 985, 348-355.

Edwards J, Jackson HJ, Pattison PE (2002). Emotion recognition via facial expression and affective prosody in schizophrenia: a methodological review. Clinical Psychology Review 22, 789-832.

Fine C, Lumsden J, Blair RJR (2001). Dissociation between 'theory of mind' and executive functions in a patient with early left amygdala damage. Brain 124, 287-298.

Gallagher HL, Frith CD (2003). Functional imaging of 'theory of mind'. Trends in Cognitive Sciences 7, 77-83.
Gallese V, Keysers C, Rizzolatti G (2004). A unifying view of the basis of social cognition. Trends in Cognitive Sciences $\mathbf{8}$, 396-403.

Hall J, Harris JM, Sprengelmeyer R, Sprengelmeyer A, Young AW, Santos IM, Johnstone EC, Lawrie SM (2004). Social cognition and face processing in schizophrenia. British Journal of Psychiatry 185, 169-170.

Haxby JV, Hoffman EA, Gobbini MI (2000). The distributed human neural system for face perception. Trends in Cognitive Sciences 4, 223-233.

Haxby JV, Hoffman EA, Gobbini MI (2002). Human neural systems for face recognition and social communication Biological Psychiatry 51, 59-67.

Hobson RP, Ouston J, Lee A (1988). What's in a face? The case of autism. British Journal of Psychology 79, 441-453.

Howard MA, Cowell PE, Boucher J, Broks P, Mayes A, Farrant A, Roberts N (2000). Convergent neuroanatomical and behavioural evidence of an amygdala hypothesis of autism. Neuroreport 11, 2931-2935.

Kim J-W, Kim J-J, Jeong BS, Ki SW, Im D-M, Lee SJ, Lee HS (2005). Neural mechanism for judging the appropriateness of facial affect. Cognitive Brain Research 25, 659-667.

Kling AS, Brothers L (1992). The amygdala and social behaviour. In The Amygdala: Neurobiological Aspects of Emotion, Memory and Mental Dysfunction (ed. J. Aggleton), pp. 353-377. Wiley-Liss: New York.

Mah L, Arnold MC, Grafman J (2004). Impairment of social perception associated with lesions of the prefrontal cortex. American Journal of Psychiatry 161, 1247-1255.

Maldjian JA, Laurienti PJ, Kraft RA, Burdette JH (2003). An automated method for neuroanatomic and cytoarchitectonic atlas-based interrogation of fMRI data sets. NeuroImage 19, 1233-1239.

Mandal MK, Pandey R, Prasad AB (1998). Facial expressions of emotions and schizophrenia: a review. Schizophrenia Bulletin 24, 399-412.

Marwick K, Hall J (2008). Social cognition in schizophrenia: a review of face processing. British Medical Bulletin 88, 43-58.

Morris JS, Frith CD, Perrett DI, Rowland D, Young AW, Calder AJ, Dolan RJ (1996). A differential neural response in the human amygdala to fearful and happy facial expressions. Nature 383, 812-815.

Nichols T, Brett M, Andersson J, Wager T, Poline J-B (2005). Valid conjunction inference with the minimum statistic. NeuroImage 25, 653-660.

Pelphrey KA, Sasson NA, Reznick JS, Paul G, Goldman BD, Piven J (2002). Visual scanning of faces in autism. Journal of Autism and Developmental Disorders 32, 249-261.

Philip RCM, Whalley HC, Stanfield AC, Sprengelmeyer R, Santos I, Young AW, Atkinson AP, Dittrich WH, Calder AJ, Johnstone EC, Lawrie SM, Hall J (in press) Deficits in facial, body movement and vocal emotional processing in autistic spectrum disorder. Psychological Medicine.

Pinkham AE, Hopfinger JB, Pelphrey KA, Piven J, Penn DL (2008). Neural bases for impaired social cognition in schizophrenia and autism spectrum disorders. Schizophrenia Research 99, 164-175. 
Pinkham AE, Penn DL, Perkins DO, Lieberman J (2003). Implications for the neural basis of social cognition for the study of schizophrenia. American Journal of Psychiatry 160, 815-824.

Rizzolatti G, Craighero L (2004). The mirror-neuron system. Annual Review of Neuroscience 27, 169-192.

Russell TA, Rubia K, Bullmore ET, Soni W, Suckling J, Brammer MJ, Simmons A, Williams SCR, Sharma T (2000). Exploring the social brain in schizophrenia: left prefrontal underactivation during mental state attribution. American Journal of Psychiatry 157, 2040-2042.

Santos I (2003). Perception of social characteristics from faces. PhD thesis, University of York, UK.

Santos I, Young AW (2008). Effects of inversion and negation on social inferences from faces. Perception 37, 1061-1078.

Schmahmann JD (2004). Disorders of the cerebellum: ataxia, dysmetria of thought, and the cerebellar cognitive affective syndrome. Journal of Neuropsychiatry and Clinical Neurosciences 16, 367-378.

Schmahmann JD, Sherman JC (1998). The cerebellar cognitive affective syndrome. Brain 121, 561-579.

Shaw P, Bramham J, Lawrence EJ, Morris R, Baron-Cohen S, David AS (2005). Differential effects of lesions of the amygdala and prefrontal cortex on recognizing facial expressions of complex emotions. Journal of Cognitive Neuroscience 17, 1410-1419.
Singer T, Kiebel SJ, Winston JS, Dolan RJ, Frith CD (2004). Brain responses to the acquired moral status of faces. Neuron 41, 653-662.

Steele JD, Lawrie SM (2004). Segregation of cognitive and emotional function in the prefrontal cortex: a stereotactic meta-analysis. NeuroImage 21, 868-875.

Stone VE, Baron-Cohen S, Calder A, Keane J, Young A (2003). Acquired theory of mind impairments in individuals with bilateral amygdala lesions.

Neuropsychologia 41, 209-220.

Tzourio-Mazoyer N, Landeau B, Papathanassiou D, Crivello F, Etard O, Delcroix N, Mazoyer B, Joliot M (2002). Automated anatomical labeling of activations in SPM using a macroscopic anatomical parcellation of the MNI MRI single-subject brain. NeuroImage 15, 273-289.

Vollm BA, Taylor ANW, Richardson P, Corcoran R, Stirling J, McKie S, Deakin JFW, Elliott R (2006). Neuronal correlates of theory of mind and empathy: a functional magnetic resonance imaging study in a nonverbal task. NeuroImage 29, 90-98.

Winston JS, O'Doherty J, Kilner JM, Perrett DI, Dolan RJ (2007). Brain systems for assessing facial attractiveness. Neuropsychologia 45, 195-206.

Winston JS, Strange BA, O'Doherty J, Dolan RJ (2002). Automatic and intentional brain responses during evaluation of trustworthiness of faces. Nature Neuroscience 5, 277-283. 